\title{
Der Stufenbau der Natur im Weltbild des Petrus Hispanus*
}

\author{
Von Heinrich Schipperges
}

Um die Mitte des 13. Jahrhunderts hatte die Schule von Siena ihr «studium generale» eröffnet. Einer der ersten Magister der Medizin war Petrus Hispanus oder Lusitanus, der hier zu einem der fruchtbarsten Schriftsteller der Hochscholastik wurde. In einem knappen Jahrzehnt sind hier in Siena, wie erst jüngere Untersuchungen ergeben haben, etwa vierzig umfangreiche Werke entstanden, meist medizinischen oder naturphilosophischen Inhaltes. Bekannt geworden sind davon nur zwei: die Summulae logicales, für 300 Jahre das Lehrbuch der Philosophie an europäischen Universitäten $^{1}$, sowie der Thesaurus pauperum ${ }^{2}$, eine zweitrangige Rezeptschrift, durch Hunderte von Handschriften und Frühdrucken erhalten, aber auch verwässert, wirklich ein schlechtes Muster für das Ingenium seines Autors.

Wer ist dieser merkwürdige Scholastiker, dessen Name in der Überlieferung der Arzneikunde wie der Logik zur Autorität wurde, dessen Schriften in ihrer ganzen naturphilosophischen Breite aber weder von den Mediävisten noch den Medizinhistorikern gelesen werden? Wie wurde er in der spätscholastischen Tradition zum «summus medicorum monarcha»? Welche historische Stellung hat Petrus Hispanus?

Wir wissen aus den Quellen, daß er nach dem Jahre 1245 aus Paris kam, wo der begabte Arztsohn aus Lissabon das Magisterium in der Philosophie und Medizin erworben hatte. Als seinen Lehrer in der Naturkunde kennen wir Albertus Magnus, als seine Meister in der Logik Wilhelm von Shyreswood, gen. de Montibus sowie den Pariser Summulator Lambert von Auxerre. Medizinstudien in Montpellier und Salerno sind sehr wahrscheinlich, aber nicht direkt dokumentiert. Dagegen erweist sich Petrus

* Nach einem Vortrag auf der Henry-E.-Sigerist-Konferenz am 26. März 1960 in Luzern.

${ }^{1}$ Grabmann (1956) 247 nennt die Summulae «das einflußreichste Lehrbuch der Logik, das es je gegeben hat». Scholz (1946) findet in ihnen zum erstenmal «eine zusammenhängende planmäßige logische Analyse der Sprache», eine Analysis von der Art, wie sie uns in Bolzanos Wissenschaftslehre erst wieder begegne (Philos. Jb. 56 [1946] 111).

${ }^{2}$ Die beste moderne Übersicht über Handschriften und Frühdrucke bietet die Bibliografia Geral Portuguesa, Vol. II. Lisboa 1944. - Vgl. auch ThовNdike II, 514-516.

${ }^{3}$ Der Cod. Harleianus 5218 BM London enthält eine «Epistola magistri Petri Hyspani ad imperatorem Fridericum super regimen sanitatis». 
in frühen Handschriften als ein Schüler des syrischen Arztes Theodonus Physicus, der am Hofe des Hohenstaufen in Palermo wirkte ${ }^{3}$.

Die weitere Laufbahn dieses Arztphilosophen ist abenteuerlich genug. Als Leibarzt einflußreicher Kardinäle, wie ОттовоNus Fieschi, des späteren Papstes Hadrian V., und Archiater beim Papst Gregor X. ersteigt Petrus rasch die Kurienleiter, wird Erzbischof von Braga und Tusculum und im Herbst des Jahres 1276 Papst Johannes XXI. - «Johannes LUSITANUS », wie die Grabschrift sagt. Mit der theoretischen wie praktischen Makrobiotik vertraut, versprach er sich einen weiten Aktionskreis, wie die Unionsbestrebungen mit der Ostkirche und Pläne zur Studienreform ${ }^{4}$ zeigen. Ein tragischer Tod machte sein Lebenswerk zunichte. Petrus starb bereits im Mai des folgenden Jahres nach Einsturz einer Decke in der Bibliothek seines Palastes zu Viterbo. Dante hat ihn bekanntlich im 12 . Gesang seines Paradieses vorgestellt, in der Gesellschaft des Hugo von Sт. VIктоR und mit seinen zwölf Büchern, das sind die «dodici libelli» der Logik ${ }^{5}$.

Aus dem literarischen Werk des Petrus greifen wir nicht das medizinische Schrifttum heraus, auf das schon SudHoff (1934) aufmerksam gemacht hatte, auch nicht die umfangreichen Opera medica, die noch unbearbeitet in der Biblioteca Nacional zu Madrid ruhen, wir beziehen uns vielmehr auf die voluminösen Beiträge zur aristotelischen Physiologie, auf die Grabmann mehrfach hinwies, ehe sie von Alonso in drei Bänden (1941 bis 1952) ediert wurden ${ }^{6}$; aus ihnen holen wir das heraus, was nicht explicit gegeben wurde, nämlich die Naturphilosophie.

Jede Rehabilitierung der Naturphilosophie muß befremdlich erscheinen, wo wir gewohnt sind, als die großen Epochen der Heilkunde die naturwissenschaftlichen Eroberungen anzusehen: die alexandrinische Grundlegung

${ }^{4}$ Johannes XXI. hat die Aristoteles-Untersuchungen inauguriert, die dann von dem Pariser Bischof StePhan TeMPIER selbständig weitergeführt wurden und zu den berüchtigten Pariser Aristoteles-Verboten (1277) geführt haben.

${ }^{5}$ Die moderne Edition bringt die Logik mit den zwölf Büchern der Handschriften, während die Frühdrucke meist die Einteilung in sieben Bücher bevorzugt haben. DaNte spricht von «Pietro Ispano», den die deutschen Übersetzungen zu «Spaniens Peter» oder einem «spanischen Peter» verunstaltet haben.

${ }^{6}$ Wir zitieren im folgenden nach diesen Ausgaben, als Scient. das Hauptwerk: Scientia libri de anima, Ed. 1941; als Quaest. den ausführlichen Kommentar zu De anima, Ed. 1944, 59-784; als Expos. die Translatio vetus libri de anima cum expositione Petri Hispani, Ed. 1952, 87-401, als Comm. den Kommentar zu «De longitudine ac brevitate vitae», Ed. 1952, 403-4.90. 
exakter Forschung, den anatomischen Durchbruch im 16. Jahrhundert, die Physiologie mit und nach HARvey, eine Zellularpathologie. Nur zaghaft und kaum systematisch wagt man sich an die Bemühungen des menschlichen Geistes um das einigende Band: an die Konzeptionen der Vorsokratiker, an die Wissenschaftstheorie der arabischen Arztphilosophen, an die Naturphilosophie der Schulen von Chartres. Die Frage nach einer Naturphilosophie der Scholastik ist im Ernst noch gar nicht gestellt worden. Dabei ist doch das, was uns diese Scholastik wie Mittelalter überhaupt so fesselnd macht, nur am Rande das Reich der Materialien. Auf der Suche hiernach sind die Wissenschaftshistoriker des vergangenen Jahrhunderts gründlich genarrt und mit Recht hinters Licht geführt worden, da sie ihren Gegenstand verfehlt hatten. Es sind nicht die Realien, die wir hier zu suchen und zu kritisieren hätten, es ist das Formale und Methodische, was uns interessiert, die Denkstruktur. Es ist die Stufenordnung des Denkens, die Methodologie, die dann selbstverständlich auch zu einer Rangordnung der Stoffe führt, zu einer Wissenschaftstheorie.

Hier aber ist Mittelalter wirklich noch ein Mittleres und ein Mittler. Hier wird es zu jenem paradigmatischen Fokus und Brennpunkt, der uns die alte Welt heller spiegelt, aber auch einen weitvorausleuchtenden Schein gibt auf den wirren Weg der Entwicklung zur Neuzeit. Es werden keine Erkenntnisse resultieren, die unserem konkreten und fortschrittlichen Weltbilde eingefügt werden könnten, wenn wir nunmehr versuchen, einmal den scholastischen Weg des Verfahrens in einer Wissenschaft einzuhalten.

\section{Zur Methodik des Scholastikers}

In der ersten Hälfte des 13. Jahrhunderts emanzipiert sich die alte scholastische Form der Quaestio, indem sie sich mehr und mehr löst vom Textbuch, das bald nur noch als Folie dient. Charakteristisch wird dieser Vorgang beim Argumentieren eines Siger von Brabant, bei Martin und Boetius von Dacien, bei Ägidius von Orléans ${ }^{7}$ - so auch bei Petrus Hispanus.

Jede «Quaestio» als Träger des Problems hat ihr «pro» und «contra» in sich. «Dictio» und «oppositio» distinguieren die Fragen und gruppieren die Antworten in eine Ordnung. Jede These der «quaestio» wird mit «rationes» versehen, je nach der Dignität des Problems. Nunmehr erhält

${ }^{7}$ Vgl. Martin Grabmann (1936) 249. 
der Opponent die Möglichkeit, innerhalb solchen diskursiven Verfahrens seine «rationes» zu plazieren. Dies nun ist Aufgabe des Schülers. Er ist es folglich, der jedem Disput das Niveau gibt und die Dimension auslotet, in der die Diskussion verlaufen soll. Dann erst tritt der Meister auf die dialektische Bühne mit seiner «solutio», die uns oft zu apodiktisch erscheint, die indes alles andere sein will als eine Endlösung mit wissenschaftlicher Stringenz. Zu oft lesen wir einleitend zur «solutio»: «multiplex est intensio circa istam quaestionem »! «Solutio » ist keine Auflösung der Fragengruppe, sondern eher das Gehege, in dem der Meister die dialektische Jagd abbläst und das Wild auffängt, gleichzeitig aber auch Trophäen verteilt, indem er die Treffer der Argumente rangiert.

Nur wenn man in dieser Weise die Partner des Dialogs mitnimmt in die dürre Dialektik der rationellen Systeme, wird man gewahr, was Scholastik eigentlich ist: immer nur Schulbetrieb, «universitas» einer wissenschaftlichen «conversatio», und in diesem gelehrten Treiben Spiel zwischen der Antwort und immer neuen Fragen, ein Spiel, das erst offen macht für das Fragwürdige im Ganzen der Welt. Um dieses Ganzen willen wandeln sich die Aspekte und wechseln mit jeden neuen «dicendum est» und «nos autem dicimus».

Bei allem Reichtum an perspektivischen Möglichkeiten gibt es im Prinzip doch nur zwei Wege für unseren Scholastiker, die er als «via experimenti» und als «via rationis» bezeichnet. Beide wollen letztlich ausgewogen und abgemessen sein. Diskursives Vorgehen ohne sinnliche Erfahrung ist absurd. Ebenso undenkbar ist Empirie ohne rationales Fundament. Auf dem wissenschaftlichen Weg garantiert erst die Vernunft die Erfahrung, indem sie ihren Weg respektiert, zurückgehend bedenkt. Deshalb heißt es bei Petrus HisPanus zunächst: «experimentum ostendit» - die Erfahrung gibt uns genügend Hinweise, aber das diskursive Vorgehen zeigt und lehrt uns doch noch ein weiteres: «quia universalior est et certificans viam experimenti»!

\section{Der empirische Schichtenbau der Natur}

Wie baut sich die Natur vor uns auf, wenn wir zunächst einmal «via experimenti» verfahren? In bestürzender Unmittelbarkeit drängt sich der elementaren Natur (natura compacta) die belebte Welt auf als eine «coagulatio debilis»: Pflanzen und Tiere, auch der Mensch, bilden ein solches Reich, das zu seiner Erhaltung eines besonderen Prinzips bedarf: «regens et dirigens ipsa». Dieser Dirigent kann die Natur nicht sein, wie wir am 
Schicksal des Schnees erfahren; etwas Neues ist hinzugetreten, das wir Seele nennen ${ }^{8}$.

Vor dem Auge unserer Erfahrung entfaltet sich unmittelbar die unverrückbar einschichtige Basis, wie sie Aristoteles in der Meteorologica behandelt hatte, in eine Welt voller Leben. Dabei ist das Vegetativum gewissermaßen noch eingeborgen in diese leblose Natur, es wird verborgen gehalten (occulta est); es ist die stumme Innenseite, die sich ausfächert, um sich in aller Stille zu repräsentieren. Offenkundig wird dieses Leben erst im Sensitivum, das mit Organen zutage tritt, manifest wird, indem es seine Werkzeuge auf einen Sinn auslegt. Vollkommen erscheint das Leben im Intellectivum, das sich selber bewußt wird, so Sinn trägt und Leben führt, indem es die Welt erfährt.

Dies alles klingt - weil wörtlich genommen - merkwürdig modern, wie auch der Grundgedanke, daß keine dieser Schichten isoliert zu denken sei. Sie durchdringen und dienen einander. Sie fordern zum Vergleich und zur Kompensation heraus. Sie erfordern eine «collatio viventium ad invicem» ${ }^{9}$. Biologie kann nie Fach werden, sie bleibt - auf den Weg der Analogie verwiesen - übergreifende, vergleichende Wissenschaft.

Die Pflanze nun - stabil, regenerationsfähig, reizarm, darin irdischer, natürlicher, kraftvoller - ist das primitive Modell für Leben. Ihr spärliches «humidum» garantiert ein stabileres Gegengewicht zur «siccitas terrestris». Mit ihrer «coagulatio fortis» steht sie machtvoll der «dissolutio corruptionis» entgegen ${ }^{10}$. Daher die souveräne «regulatio partium»: Abstoßen des Alten und üppige Regeneration aus einem "principium productionis» heraus; daher ihre "generatio multiplex». Nahrung und Wachstum bleiben stabil, nicht zu stark gehindert durch Reize. Daher - «absque sensibilium impedimento libere operantes» - erreichen sie jene beachtliche «fortitudo virtutum naturalium ${ }^{11}$. Sie haben Seele, aber - weder Spezies noch Individuum - nur als eine einzige, bleibende Form dieses ganzen Reiches ${ }^{12}$.

${ }^{8}$ Quaest. 503, 2: per nexum elementorum non conservantur, sed per aliud principium. Hoc autem est anima, quae per sui praesentiam ipsa conservat.

${ }^{9}$ Hierzu ausführlich in der Expositio zum Traktat De longitudine ac brevitate vitae IV; Ed. Alonso 1952, 475 ss. - cf. Scient. 89 : Habent autem ad invicem necessitatem ordinis; l. c. 88 : Est autem inter ipsas ordo naturalis.

${ }^{10}$ Comm. III, 6: Unde non habent humiditatem facile eliquabilem nec dissolubilem, sicut animalia.

${ }^{11}$ l.c.: fortitudo virtutum naturalium circa nutrimentum et augmentum operantium, quae in eis fortes inducunt operationes absque sensibilium impedimento libere operantes.

$12 . .$. in utrisque anima una in multas multiplicatur. 
Das Tier ist demgegenüber durch seine Sinnlichkeit gezeichnet, die ihm seine instinktive Sicherheit verleiht, es dafür aber auch um so sicherer seiner Umwelt verhaftet, es ins Milieu verankert. Den « animalia imperfecta » fehlt die Sinnlichkeit per Distanz, wie Petrus sagt. Die Tiere unterstehen der puren Aktualität, gefesselt an Ort, da, ohne Zeit. Ihnen fehlt noch jene Emotion, der «motus localis», der übers Werkzeug, die «organa», an ein erstrebtes Ziel trägt. Solche Emotion ermöglicht erst die sinnliche Abstimmung auf ein gemeinsames Ziel. Somit fehlt hier die Freiheit, besser noch: die Befreiung (deliberatio), wie sie mit der Distanzierung gegeben ist, ein Freiwerden, wie es die Vergangenheit bedingt, ohne die ja auch des Menschen Existenz auf die Dimension des Animalischen zusammenschnurren würde ${ }^{13}$.

Der Mensch endlich, «via experimenti» betrachtet, ist ebenfalls ein biologisches Phänomen. Auch er steht auf der Basis des Vegetativen und lebt im Milieu des Sensitiven. Auch sein Gesetz heißt Wachstum und Zeugung. Dabei erweist er sich als das biologische Mangelwesen, eine «mollis coagulatio» oder «coagulatio levis», eine «debilis coagulatio». Nur in dieser Verfassung wird er leichter ernährt, aber auch empfänglicher für sinnliche Eindrücke. Er wird passibel im höchsten Grade, vulnerabel, er hat am meisten Anlage zum Sterben. Zwar wird das labile Vegetativum kompensiert durch ein hochkompliziertes Sensitivum, aber gerade dies bringt neue Spannung, mehr noch, einen Kampf, dessen Ausgang nie ungewiß bleibt: Sieger ist im Lebenskampfe aus physiologischen Gründen immer der Tod, die «victoria caloris» über die Lebensfeuchte, das «pabulum vitae» ${ }^{14}$.

Diese Schwäche des Menschen wird in der eigenartig schwebenden Stufenordnung und in einer ineinandergleitenden Verkehrung der Rangfolge zum Vorzug. Im Menschen nämlich repräsentiert sich erst der ganze Stufenbau der Natur, weil er ist die «regula viventium ${ }^{15}$, das biologische Prinzip. Durch seine Stellung wird die Stufung auch in den anderen Lebewesen erkannt und so herunter bis zu den unvollkommenen und gleichsam verbor-

${ }^{13}$ Expos. 376, 21: non sentiunt per distantiam sed omne quod est eis appetibile actualiter est eis coniunctum secundum locum, et propterea non debetur eis motus localis ad consequendum sensatum desideratum. - 377, 30: Virtus autem deliberativa inest ratiocinativis, scilicet, ad quam consequitur appetitus intellectualis. Quod autem deliberatio sit in rationalibus, ostendit, quia considerare utrum sit agendum hoc aut hoc, est opus rationis, sed huiusmodi opus est deliberatio; ergo deliberatio est in rationalibus.

14 Hierüber ausführlich in Comm. I, 2.

15 Scient. 541: Haec autem distinctio in homine, qui omnium est regula viventium, certior reperitur et per ipsum in aliis perfectis et per haec in imperfectis animalibus cognoscantur. 
genen Wesen. Selbst im Pflanzenreiche zeigen sich bestimmte Teile vergleichbar dem tierischen Organ, Teile, die dann in diesem Organismus zum. «fundamentum regiminis» werden ${ }^{16}$. Der Vergleich bringt auch hier die Sonderung mit sich. Die «aequalitas» im «temperamentum» etwa, die «harmonia vitae» innerhalb der «contrarietas complexionum», alles das gilt - per analogiam - für jedes Lebewesen, es gilt besonders für das Tierreich, am meisten aber gewinnt es Gültigkeit, als eine Spielregel oder als das Naturgesetz, beim Menschen: «quod est perfectius exemplar.» ${ }^{17}$

Bis hierhin vermögen die Betrachtungen zu kommen, die einer vergleichenden Biologie auf empirischer Basis erwachsen, wobei kaum noch zu betonen ist, daß hier "experimentum» nie so etwas meint wie Versuch, also Experiment, sondern immer nur das Verfahren des Erfahrens selber; es will mehr den Eindruck zum Ausdruck bringen, jene Weltoffenheit der Aufmerksamkeit bei aller Anschauung und Beobachtung des gesunden Menschenverstandes, mehr nicht! Schärfer allerdings wird diese beobachtete Ordnung und der Stufenbau der Natur durch ein Phänomen akzentuiert, das an der Grenze der Erfahrbarkeit liegt, das nicht mehr dem «experimentum» unterliegen kann. Die Erfahrung zeitigt nämlich in allen biologischen Bereichen, am einschneidendsten beim Menschen, eine Erscheinung, die nicht mehr durch die «via experimenti» gedeckt wird - und das ist das Phänomen der Zeit.

Es ist nicht so modern, wie es den Anschein hat, aber es ist recht konsequent gedacht, wenn Petrus Hispanus dies auf eine lapidare Formel bringt: "tempus est causa corruptionis. ${ }^{18}$ Natürlich: Leben zeitigt den Tod - das ist eine alte Erfahrung! Aber es will doch in seiner ganzen Bedenklichkeit auch rational durchdacht sein, wenn yon Bios und Natur, von Welt und Vergänglichkeit, wenn vom Menschen mit seinem Pathos und Sterben die Rede ist.

Was ist denn die Zeit, wenn man sie biologisch und nicht mehr physikalisch nimmt? Ist Zeit, so fragt Petrus, faßbar und damit teilbar? Ja in der

${ }^{16}$ Scient. 541: In plantis vero quaedam partes animalium membris sunt proportionales, quae in ipsis sunt regiminis fundamentum.

17 Auch dieser Gedankengang wird am geschlossensten in Comm. 463 ausgeführt: ... in omnibus viventibus proportionaliter sunt intelligendae, ut in homine quod est perfectius exemplar, disponitur; et per ipsum in perfectis aliis, per perfecta in imperfectis, et per animalia in plantis causae omnes aut quaedam secundum analogiam significantur.

${ }^{18}$ Comm. 442: Tempus igitur causa corruptionis dicitur, quia rerum corruptio sub tempore accidit, omnes enim res quaecumque sunt in tempore, cum tempore labefiunt et corrumpuntur. 
Möglichkeit, in Wirklichkeit aber, «in actu», nie und nimmer. Die Stücke der Zeit entsprechen keiner räumlichen Länge. Zeit duldet kein geometrisches Verständnis. Die Korruption als «occursus rerum in tempore» ist auf dem Wege der Empirie nicht zu klären ${ }^{19}$. Das Wesen des «homo patiens» mit seinem «quasi naturalis»-Tod muß auf einem anderen Wege gesucht werden, der «via rationis», wobei mit der Spekulation auch der ganze Raum der historischen Überlieferung, die Tradition der «auctores», gegeben ist. Dieses Sein in der Zeit genügt, um die Geschichte als ein unumgängliches Kriterium und Korrektiv für jede Anthropologie zu deklarieren und zu postulieren; ohne diese «Zeit» wäre die Medizin empirisch faßbar, «via experimenti», sie wäre Naturwissenschaft. Wir müssen den ganzen Weg noch einmal gehen, ein zweites Mal den Stufenbau der Natur aus der Sicht des Scholastikers entwerfen, um zu einem geschlossenen und lückenlosen Weltbild, zu einem Sein mit Zeit, zu kommen.

\section{Zur spekulativen Physiologie der Scholastik}

Es fällt uns schwer, auf dem spekulativen Wege der scholastischen Methode mitzuhalten, all die schwerfälligen dialektischen Sprünge mitzumachen, immer wieder diese scheinbaren Tautologien nachzuvollziehen; aber nur, wenn man sich dieser Mühe, solchem scholastischen Training, unterzieht, wird man begreifen, warum dem Scholastiker eine Deutung der Außenwelt von den Realwissenschaften aus nicht genügt, warum er nunmehr die Welt der lebendigen Erscheinungen auch noch von der diskursiven Deduktion aus, also «via rationis», untersuchen will.

Auch in diesem Aspekt werden wir wieder auf die eigentümliche Bewegtheit dieser Stufenleiter aufmerksam werden. Das Einfache wird Muster der Vollkommenheit, das Höhere erweist sich als abhängig vom tragenden Grund, die mittleren Schichten zeigen eine paradoxe Spiegelung dieses vielschichtig abhängigen Weltbildes. Wachstum wird Leitbild der geistigen Perfektion, Zeugung ist exemplarisch für sittliches Heil, beide im Sinne des Ganzen und Heilen, der gesunden Integrität, dessen, was die Araber mit «salam» bezeichnet hatten. Natur bekommt in jedem Detail Akzente des Wertes. Die rationale Vertiefung eröffnet uferlose Horizonte, und dies von jeder Stufe des Schichtenbaues aus.

Wir wählen als einfaches Beispiel die vegetative Schicht und verfolgen ihre Bahnen «via rationis».

${ }^{19}$ Dieser «occursus» fungiert als Leitthema in De longitudine II. 
Petrus bedient sich in der Diskussion des traditionellen Schemas. Innerhalb der "virtutes vegetabiles» garantiert die «virtus nutritiva» die Permanenz der «integritas perfecta». Zum Leitbild dieser Perfektion wird die «virtus augmentativa». Eine «virtus generativa» überwacht die «integritas speciei». Wir begnügen uns, einer Spur in das dialektische Gestrüpp zu folgen, der «virtus nutritiva».

Sie verzweigt sich vierfältig. Eine «virtus praeparativa» der Vorbereitungsphase nimmt Nährstoffe auf und verteilt sie. Unter der «virtus comprehensiva » werden die Nahrungsstoffe gleichsam herausgelockt und in die Obhut des Organs gegeben ${ }^{20}$. Dann erst kommt die "virtus applicativa» zum Zuge: die Nahrung wird einverleibt, mit den Einzelteilen der Gewebe verknüpft. Die «virtus assimilativa» beschließt die Phase dieser Konformation ${ }^{21}$. Nunmehr steht die Nahrung den «dispositiones accidentales», der situationsbedingten Funktion, zur Verfügung.

Im Dickicht der Dialektik könnte man so nach allen Richtungen weitergehen; wir nehmen nur einen Faden zur Hand und verfolgen die Spur der "virtus praeparativa». Sie führt auf fünf verschiedene Pfade: Appetit bestimmt das Bedürfnis. Es ist jene "virtus appetativa», eine Konkupiszenz, die ausreicht, das «detrimentum» auszugleichen und die somit Satisfaktion schafft im biologischen Gleichgewicht. Nunmehr wird die "virtus attractiva» den Außenstoff umstimmen und auf eine «similitudo» hinlenken. Ein retardierendes Moment, die "virtus retentiva», bremst diese Attraktion, fixiert die «actio conversionis» und ermöglicht damit die allmähliche Einverleibung ${ }^{22}$. Die Assimilation selbst wird endlich vorbereitet durch die "virtus digestiva», die erst einer "virtus expulsiva» den Weg freimacht, das Schädliche als überflüssig auszuscheiden.

Wir gehen noch einen letzten Schritt, in einer-abermals spezialisierten Dimension und wiederum nur am Beispiel, der «virtus digestiva». Sie ist es, die die «massa chylosa» umstimmen kann zur Abgabe an das Blut. Sie macht aus der "massa chylosa» eine "massa chimosa», das ist schon die Blutsubstanz, die alsdann in die «ultima humiditas» verwandelt werden

20 Scient. 93: Praeparativa est virtus nutrimentum transmutans ac disponens ut menbris habile effici mereatur. Comprehensiva est virtus nutrimentum alliciens et menbris coniungens.

21 Wir bedienen uns hier und im folgenden möglichst genau der Termini des Perrus.

22 Scient. 94: Retentiva vero est virtus nutrimentum stabiliter retinens ut conversionis actio in eo fixa permaneat, quousque completam praeparationem suscipiat ac menbrorum substantiae conformetur. 
muß ${ }^{23}$. Auf diese Weise gleitet die «virtus digestiva » sanft und reibungslos hinüber in eine "assimilatio completa».

Das ist der Weg, und so läuft die Methode durchgehend auch über die übrigen Felder. Das ist Scholastik. Da wird nichts Neues erforscht, aber darauf kommt es auch gar nicht an. Man hat genug über diese Scholastik gespottet ${ }^{24}$, aber im Ernst : Ein Schüler, der diese Gedankengänge mitgemacht hat, der nicht nur einer Verzweigung, sondern dem ganzen Geflecht nachgegangen ist, der diese komplizierten Verhältnisse schulmäßig durchmeditieren mußte, der - wie bei Petrus ausgiebig vordemonstriert wird - das Ganze mit «quaestio» und «oppositio» und «solutio» durchexerzieren mußte, sollte ein solcher Schüler ein blasseres Bild von den physiologischen Problemen gehabt haben als etwa unsere Physikumskandidaten?

In derselben Manier wie das Vegetativum wird das Sensitivum durchgespielt, durchexerziert, durchrationalisiert. Hier nur das Ergebnis mit seiner anthropologischen Relevanz. Die Sinne sind Pfade zu Freud und Schmerz, abermals in strenger Rangordnung der Reizstufen und Schmerzqualitäten. Das Sensorium bedient sich seiner «organa» als Werkzeuge zu einem Ziel. Hieraus versteht sich die Reibung, der Verschleiß, alles Wegbereiter des Sterbens. Im Tod wird der jeweilige Nexus gelöst ${ }^{25}$. Tod als solcher kann nur negativ interpretiert werden. Was aber ist das Positivum, der rätselhafte Nexus selber?

Petrus geht hier auf die geheimnisvolle «unio animae cum corpore» ein, die sich im vegetativen Bereiche leichter erklären lasse, schwieriger schon bei sensiblen Wesen, am schwersten beim Intellectivum ${ }^{26}$. Unter Verzicht auf jede Lokalisation im Somatischen ${ }^{27}$ werden vermittelnde Medien,

${ }^{23} \ldots$ in chimosam ac sanguineam transducens ut ad humiditatem ultimam perveniat.

${ }^{24}$ So Kantorowicz etwa über die auf einer Nadelspitze tanzenden Engel. Hierzu äußert sich Grabmann (1936) 106: «Die große Scholastik des 13. Jahrhunderts trägt in ihrem geistigen Antlitz dermaßen die Züge wissenschaftlichen Ernstes, daß solche Vorwürfe nicht zutreffen.»

${ }^{25}$ Die Lösung wird mit Termini umschrieben wie: privatio, dissolutio, destructio, dissipatio, ruptio, corruptio, regressio, consumptio, extinctio.

${ }^{26}$ Scienti 71: Unio igitur substantiae praecedit virtutem unionem, et ideo virtutes non sunt prima media huius unionis, sed alia prima antecedit. Es sind folglich die «media», die stufenweise das Funktionsgefüge erklären. Deshalb wird die Vereinigung bei den höheren Lebewesen besonders schwer verständlich. $l$. c. 73 : Intellectiva vero cum a natura corporali maxime seiungatur, humano corpori mediis altioribus copulatur.

${ }^{27}$ Expos. 375, 3: quod anima in corpore est ubique tota; ergo habet locum corporis per accidens ... 
«media iuncturae», herangezogen, die jedoch gerade nicht spiritualistisch zu deuten sind, sondern im Leiblichen verwurzelt sein müssen ${ }^{28}$. Es sind dies vor allem die «spiritus » in Analogie zu einem «lux spiritualis » und der «calor naturalis» als Abbild eines «calor spiritualis». Eine besondere Untersuchung der Psychiatrie des Petrus Hispanus erst könnte erweisen, wie sehr sich hier der Scholastiker als ein Somatiker erweist. Hierbei scheut er sich nicht, auch die heißen Eisen der Überlieferung anzufassen, so den Traktat des Galen: «Daß die Vermögen der Seele Folgen der Mischungen des Körpers sind ${ }^{29}$. Hier wird dem Galen Augustinus gegenübergehalten - auch dies ein reizvolles Thema! Die leib-seelische Problematik hält Petrus für äußerst diffizil; dies spürt man, wenn er in seiner schwerfälligen Argumentation plötzlich innehält und ausruft: «Ganz wunderbar aber muß uns die Anordnung der Natur erscheinen bei einer derartigen Vereinigung ! $^{30}$

Als genau so wundervoll imponiert ihm das System der Abhängigkeiten: daß Intellekt sich stützt auf Sinnlichkeit, daß Sinne abhängen vom Organ, daß die Verhältnisse des Organismus wurzeln im Elementaren! Auch hieraus ergeben sich weittragende Konsequenzen für eine scholastische Ekenntnistheorie, wenn wir lesen: «sensus recipit elementa» und «intellectus sequitur sensum ${ }^{31}$. An diesem Fragenkreis offenbart sich das Naturgesetz dem Menschenverstand als eine Art von immanenter Vorsehung ${ }^{32}$. Wer aber - so dringt der Scholastiker weiter - wer regiert diese «necessitas ordinis ad invicem»? Wo liegt das regulierende Prinzip dieser «machina corporis»? Wer leitet das «regimen» an zu seinem Reglement? Nur eine weltweite Ordnung dienender Kräfte kann hier dem Verständnis helfen. Die vegetative Schicht dient in ihrer ganzen Breite solchem «regimen»; das Sensitive instruiert den Intellekt und dient damit ebenso; die «virtutes intellectivae» aber erst regieren die sensitiven und diese die vegetativen und diese endlich die «virtutes corporales » ${ }^{33}$. So schließt sich - höchst geheimnisvoll-der Kreis.

${ }^{28}$ Scient. 376: Media iuncturae in natura corporis consistunt. Nach Avencebrol und AverROEs sind diese «Media» ein Teil der dreifachen Materie. Cf. Quaest. 510, 14: sicut habetur in libro fontis vitae triplex est materia, scilicet, materia corporalis et materia spiritualis et materia media. - Die letztere soll einem «corpus superior» angehören.

${ }^{29}$ Quaest. 450, 9: Quia secundum motum humorum moventur operationes animae ...

30 Scient. 74: Mirabilis autem apparet naturae dispositio in hac unione.

31 Diese Kernstücke einer Erkenntnistheorie fordern vor allen Dingen zu einem Vergleich mit den zeitgenössischen Summen der Hochscholastik heraus.

32 Scient. 331: Lex naturae provida virtutem motivam ab anima sensibili emanantem animali ad sui regimen disposuit ordinari. $\quad{ }^{33}$ Scient. 121: Intellectivae igitur regent sensitivas et sensitivae vegetativas et hae virtutes corporales. 


\section{Der Stufenbau in mystischer Weltschau}

Ein drittes Mal umkreist unser Scholastiker die gleichen Fragengruppen, aber jetzt suchen wir vergeblich die konsequente Methodik. Eine «via mystica» wird mit eigenen Worten nicht vorgeschlagen, aber sie tönt doch deutlich genug an. Aus dieser letzten Perspektive wird der «ordo naturalis» gleichsam von oben nur eingesehen, nur hellsichtig erblickt als ein Schichtengebäude, wie es der «divina providentia» entströmt. Diese Emanation aber mit ihren «multiformes gradus » mündet wie von selber in ein hierarchisches Weltbild, bildet die Weltanschauung dieser Hochscholastik.

Was von dieser letzten Sicht aus die gesamte Natur durchzieht, ist überraschenderweise zunächst als ein Trieb bezeichnet, ein natürlicher «appetitus permanentiae», der aus der göttlichen Vorsehung hinüberströmt in jedes Ding. Alles, was lebt, wirkt zum Heil seiner Existenz hin. So will es natürliche Ordnung. So erklärt sich der Trieb nach Dauer. Nur so erhält sich das Gesamt an Energie konstant in der Welt: «integris universi permanet ${ }^{34}$ ! Die Natur wird dabei definiert als eine eingebundene Weltkraft, die aus verwandten Wesen neue Verwandtschaft schafft ${ }^{35}$, die damit aber auch gerade den dumpfen Trieb zu einem hellen Zug werden läßt, der über die Assimilationen der Erfahrung und der Vernunft hinüberreißt in die Abgründe des Lichtes.

Hier erst klärt sich das Bau-Werk auf, Schicht um Schicht. Licht als «forma prima» ist unter allen Gebilden der Natur das edelste, auf das alles sich ordnet, um dessentwillen alles ist. «Alles Sinnenhafte wurzelt nämlich im Licht als seinem innersten Wesen. Es ist nicht selbst das Licht, ebensowenig wie der farbige Schein schon Licht genannt werden kann, aber es wurzelt im Lichten ${ }^{36}$. All unsere Leiblichkeit hat in sich solche Lichtnatur, gleichsam eingeborenes Licht, das uns hält und heilt ${ }^{37}$. Wir sind ein Abglanz

${ }^{34}$ Die Erhaltung der Weltenergie wird durch drei große Gesetzmäßigkeiten garantiert: im physikalischen Bereich durch eine «transmutatio mutua» der Elemente, im biologischen Feld durch eine «renovatio» der Säftemischung sowie durch die kontinuierliche "propagatio et multiplicatio seminis». Vgl. hierzu Comm. 445.

${ }^{35}$ Scient. 165: et secundum hunc ordinem natura operatur, quae est virtus insita rebus ex similibus similia producens. Vgl. auch $l$. c. 157: omnia propter hoc operantur, ut suam salvent existentiam, secundum quod ordo competit naturalis; ex divina enim providentia emanat appetitus permanentiae in omnem rem.

${ }^{36}$ Expos. 197, 19: omnia sensibilia radicantur in luce tanquam in ima radice, non quia ipsa sint lux, non enim debet dici quod color sit lux, sed radicatus in luce.

${ }^{37}$ Expos. 278, 23: omne corpus compositum habet in se naturam caelestem ut lucem incor- 
der höheren Welt, deren Licht im Organismus sich als Heilkraft auswirkt, eine helle Kraft, die ganz und gesund macht; wir tragen mit uns in aller Leiblichkeit den lichten Lebenskeim unseres Heils.

Dieser abendländische Beitrag zur Lichtmetaphysik, der nur mit einigen Linien skizziert werden konnte, ist nun freilich ohne die Quellen nicht zu erklären. An dieser Stelle werden wir gewahr, wie mächtig der Strom einer orientalischen Gnostik in das aristotelische Gefüge des antiken Erbes eingebrochen ist. Die begrifflichen Schemata eines Aristoteles und Galen lassen uns hier im Stich. Es ist die Tradition einer anderen Welt, die Petrus Hispanus durch die Araber kennengelernt hatte, vor allem durch die Schriften des Algazel und des Avencebrol. Zum medizinischen Dreigestirn aus IsaAC und HaLY und Avenzoar tritt hier das philosophische aus Maimonides und Avicenna und Averroes. All das wird zu kompensieren versucht durch die christliche Mystik eines Augustinus und PseudoDionysius Areopagrta ${ }^{38}$. Erst dann erklärt sich dieses Weltbild, das auf der Autorität der Antike basiert, auf den jonischen Naturphilosophen wie den Atomtheoretikern, das sich im platonischen Geiste gründet wie, über alles informierend, dem System des Aristoteles.

Aber es täuscht auch hier, wollte man mit diesen Quellen den Strom selber erklären. Die Weltanschauung des Petrus Hispanus will als Ganzes und Eigenes erkannt werden ${ }^{39}$. Alles ist originell verarbeitet und mit einer geistigen Intuition ersten Ranges an die Schüler herangetragen. Als ein solches Werk imponiert es noch heute. Von einer « Oberaufsicht der Theologie» über die Naturwissenschaft, wie DiJksterhuis dies für die Scholastik formuliert hat ${ }^{40}$, kann hier keine Rede sein. Es muß uns auch in dieser letzten Sicht bestürzen, wie sehr die persönliche Note die scholastische Strenge durchbricht, wie ein eigener Ton der begrifflichen Schematik eine

poratam, qua mediante conservatur et est forma eius particularis in sua materia ipsam perficiens et, sicut sine hac natura conservante non potest conservari compositum, similiter nec potest in aliquam operationem exire nisi per absolutionem huiusmodi virtutis.

${ }^{38}$ Der Clm 7983 (S. XIII/XIV) bringt eine «Expositio librorum beati Dyonisii a Petro yspano editum ".

${ }^{39}$ Quellen, die in reichem Maße vor allem in der Expositio fließen, fehlen vollständig in der Scientia de anima; dafür heißt es hier an einer charakteristischen Stelle auf f. $66^{\mathbf{r}}$ : in antiquorum sermonibus circa animam non ad plenum veritatis apex ac profunditas attingitur!

40 In dem ausgezeichneten Werk von E. J. DiJksterhuis: Die Mechanisierung des Weltbildes (1956) wird Petrus Hispanus nicht berücksichtigt. 
Stimmung verleiht, eine Stimmung, die aus aller Ordnung der Welt ertönt ${ }^{41}$. Aus dieser Sicht heraus will der Stufenbau der Natur nicht von unten erkannt und nicht von oben bewertet sein. Es muß uns bei diesem Wechsel der Werte in schwebender Schichtung überraschen, daß das Heil in die niederen Triebe verschlüsselt wird, wie sie uns gegeben sind mit «uti alimento» und mit «generare», und beide Male heißt es deutlich und kernig: "ut salvetur» ${ }^{42}$ ! Das Heilsverlangen wird nicht in einem spirituellen Bereiche gestillt, es quillt durch alle Schichten der Schöpfung, es strömt durch die ganze Geschichte des Menschen. So will es Natur, daß «res salvae conserventur ${ }^{43}$. Alles geht auf einem natürlichen Wege der großen Wiederherstellung entgegen, einem «status incorruptionis», einer «decoratio incorruptionis ${ }^{44}$. Dies erreicht die Natur allerdings nicht mehr aus ihrer eigenen Gesetzmäßigkeit, das wird hinzugetan «ex dono factoris excelsi» ${ }^{45}$.

\section{Zusammenfassung}

Wenn wir dieses scholastische Lehrstück über den Aufbau der Natur und ihre Schichtung zu einem Weltbild konkretisieren wollen, so finden wir kaum ein Mehr an realem Wissen. Was uns auf den weiten Feldern der griechischen und arabischen Traditionen begegnet, ist eher ein Raum an formalen Bezügen und Strukturmerkmalen des Denkens. Wir finden eine Rangordnung der Stoffe, wie sie aus einem dialektischen Spiel resultiert, einem Spiel, dessen Regeln die Erfahrung angibt und die mit den Regeln

41 E. Gilson hat diese Empfindung in seinem neuen Werk über Johannes Duns Scotus (1959) 594 präzisiert: «Man würde jedoch kaum das Interesse der Historiker und ganz und gar nicht das der Philosophen finden, wenn man ihnen sagte, daß alle diese großen Gedankengebäude, die uns das Mittelalter hinterlassen hat, im Grunde Erfindungen der Liebe waren.»

42 Espos. 149, 10: uti alimento ut salvetur per aliquod tempus in suo esse proprio; generare ut salvetur in esse perpetuo, scilicet, in simili secundum speciem.

${ }^{43}$ Comm. 446, 10: Quae autem a tempore inevitabiliter corrumpuntur per aliarum rerum ordinationem debitam conservantur, ut per ipsam occursus rerum in quibus consistit corruptio, sub tempore evitetur vel reprimatur, ut secundum certum periodum res salvae absque corrumpentium causarum impedimento in statu debito conserventur.

${ }^{44}$ Comm. 485, 6: ... destinatus, a divina providentia, quae status machinae mundi cursum finire intendit, ut ad meliorem statum perducat.

${ }^{45}$ Comm. 485, 15: Hanc autem incorruptionis decorationem non ex suarum virtutum acquirent exigentia, sed ex dono factoris excelsi; cuius enim est potestas cuncta ex nichilo condere, ab eodem virtutis emanat privilegium corruptibiles creatures sub incorruptionis protectione ad perpetuitatis statum deductas nexu constantiae indissolubili conservare. 
des Denkens in Übereinstimmung kommen. Nur eine solche vorläufige und mehr methodologisch interessierende Zeichnung sollte herausgearbeitet werden.

Was dahinter auf uns wartet, ist die völlig unbearbeitete Naturphilosophie der Scholastik, zu derer vorthomasischen Periode Petrus Hispanus den interessantesten Beitrag gegeben hat. Was wir gerade bei diesem Arztphilosophen erwarten dürfen, ist eine umfassende Theorie der Welt und vom Menschen, die in den theologischen Summen gewagt wurde, die aber in der Summa der Medizin nicht zum Tragen gekommen scheint. Ist die Aufgabe - so müssen wir fragen -, die sich Petrus Hispanus gestellt hatte, sich und mehr noch seinen Schülern, angegangen und gelöst worden? Hat sie in den folgenden Jahrhunderten eine Nachfolge gefunden? Hat sie uns etwas zu sagen, die wir immer noch auf der Suche sind nach einer theoretischen Unterbauung unserer Wissenschaft, nach einer fundierten Medizinischen Anthropologie?

Erst nach einem sachgerechten Studium an den Quellen, die jetzt in reichem Maße zuhanden sind, wird sich eine Antwort finden lassen auf die Frage nach der Bedeutung einer scholastischen Naturphilosophie für die Medizin und der Rolle, die in dieser Übergangszeit einer ihrer ersten Köpfe gespielt hat: Petrus Hispanus. 


\section{Literaturhinweise}

Berger A. M.: Die Ophthalmologie (liber de oculo) des Petrus Hispanus. München 1899.

Grabmann Martin: Ein ungedrucktes Lehrbuch der Psychologie des Petrus Hispanus im Cod. 3314 der Biblioteca nacional zu Madrid, in Gesammelte Aufsätze zur Kulturgeschichte Spaniens 1 (1928) 166-173.

- Handschriftliche Forschungen und Funde zu den philosophischen Schriften des Petrus Hispanus, des späteren Papstes Johannes XXI., in SB Bayer. Akad. Wissensch. Phil.hist. Abt. München 1936.

- Mittelalterliches Geistesleben. I-III. München 1926-1956.

KöHLer JohaNn ToBias: Vollständige Nachricht von Papst Johann XXI., welcher unter dem NamenPetrus Hispanus als eingelehrter Arzt und Weltweiser berïhmt ist. Göttingen 1760.

Mullally J. P.: The Summulae logicales of Peter of Spain. Notre Dame (Indiana) 1945.

Pedro Hispano: De anima. Lo publica y anota el P. Manuel Alonso. Madrid 1941.

- Obras filosóficas II. Comentario al «De anima» de Aristoteles. Edición P. Manued Alonso. Madrid 1944.

- Obras filosóficas III. Expositio libri de anima ... Edición P. Manuel Alonso. Madrid 1952.

Petri Hispani Summulae logicales, quas e codice manu scripto Reg. Lat. 1205 edidit I. M. BocheńsKi. Torino 1947.

Petrus Hispanus, in Bibliografia Geral Portuguesa. Vol. II. Lisboa 1944.

Riesmann David: A Phisician in the Papal Chair, in Ann. Med. Hist. 5 (1923) 291-300.

SARTon Ge0Rge: Introduction to the History of Science. II, 2. p. 889-892 (mit Literaturhinweisen).

Srmonin H.: Magister Petrus Hispanus, in Arch. Fratrum Praedicatorum 5 (1935) 340-343.

Stapper Richard: Papst Johannes XXI. Eine Monographie. Münster 1898.

Sudhoff Karl: Petrus Hispanus, richtiger Lusitanus, Professor der Medizin und Philosophie, schließlich Papst Johannes XXI., in Med. Welt (1934), 1-10.

Thorndike Lynn: A History of Magic and Experimental Science. II, 488-516.

Wilke Walter: Der Arzt Petrus Hispanus und seine Bedeutung für die Zahnheilkunde. Med. Diss., Leipzig 1924. 